\title{
Féeries
}

Études sur le conte merveilleux, XVII $-\mathrm{XIX}{ }^{\mathrm{e}}$ siècle

\section{Peau d'Âne, un conte merveilleux haut en couleur : de Perrault à Demy, des réécritures inspirées}

Peau d'Âne, a Colorful Fairy Tale: From Perrault to Demy, Inspired Rewrites

\section{Danièle Henky}

\section{(2) OpenEdition}

1 Journals

\section{Édition électronique}

URL : https://journals.openedition.org/feeries/3817

DOI : $10.4000 /$ feeries.3817

ISSN : 1957-7753

Éditeur

UGA Éditions/Université Grenoble Alpes

\section{Édition imprimée}

ISBN : 978-2-37747-327-4

ISSN : $1766-2842$

\section{Référence électronique}

Danièle Henky, "Peau d'Âne, un conte merveilleux haut en couleur : de Perrault à Demy, des réécritures inspirées », Féeries [En ligne], 17 | 2021, mis en ligne le 17 décembre 2021, consulté le 14 janvier 2023. URL : http://journals.openedition.org/feeries/3817 ; DOI : https://doi.org/10.4000/feeries.3817

Ce document a été généré automatiquement le 14 janvier 2023.

Tous droits réservés 


\title{
Peau d'Âne, un conte merveilleux haut en couleur : de Perrault à Demy, des réécritures inspirées
}

\author{
Peau d'Âne, a Colorful Fairy Tale: From Perrault to Demy, Inspired Rewrites
}

\author{
Danièle Henky
}

1 Le merveilleux est un concept complexe, en raison notamment de son ancienneté dans le discours poétologique. L'éventail des approches est large selon qu'on considère la «merveille » médiévale, les traductions des Mille et Une Nuits, les fées "à la mode » française des $\mathrm{XVII}^{\mathrm{e}}$ et $\mathrm{XVIII}{ }^{\mathrm{e}}$ siècles, la célébration du merveilleux par les surréalistes ou ses résurgences dans la fantasy contemporaine. Par-delà ces différents aspects, le merveilleux peut être envisagé comme une catégorie esthétique renvoyant à la représentation de ce qui, selon l'étymologie, provoque étonnement et admiration : à l'origine, la « merveille » saisit l'esprit parce qu'elle s'inscrit au rebours de l'expérience ordinaire, tenant du prodige, de la magie ou du miracle. De ce fait, la mise en écriture $\mathrm{du}$ conte merveilleux ne saurait se réduire à la mise en œuvre de quelques items morphologiques fondamentaux. Elle joue sur divers principes esthétiques et poétiques complexes et combinés. La formulation, le choix des mots mais aussi celui des couleurs, exprimées ou suggérées, sont des composantes fondamentales qui permettent d'en approcher la poétique.

2 L'observation de la réécriture de certains contes permet tout particulièrement de mettre en évidence les liens qu'entretiennent, par exemple, le merveilleux et la couleur par l'intermédiaire de personnages, d'un bestiaire, d'un ensemble d'objets et/ou de costumes magiques afin de stimuler l'imaginaire du lecteur en créant, au fil du récit, un univers coloré, expression à la fois du genre auquel il appartient mais aussi peut-être d'une époque et d'un style. Lorsque Jacques Demy met en scène le conte de Perrault, "Peau d'Âne ", en ayant recours, dans de nombreuses scènes du film, à une gamme chromatique très étendue sans que le conteur n'en ait fait explicitement mention dans son texte, sa lecture dévoile, d'une part, à quel point son inventivité est activée par le récit merveilleux et, d'autre part, les ressources sémantiques indicibles du texte. 
3 Nous essaierons, en analysant le conte de Perrault puis la réécriture inspirée du cinéaste qui a rendu «Peau d'Âne » universellement célèbre, de voir ce qui, dans le texte, a pu susciter, dans le film, cette mise en couleur du conte initial. Nous nous demanderons, entre autres, quelles sont les couleurs originelles citées ou évoquées par Perrault et leurs effets dans le conte, et ce qui a pu en induire d'autres, avant de tenter de cerner la symbolique et la poétique de ces couleurs dans le récit comme dans le film merveilleux.

\section{«Peau d'Âne » de Charles Perrault : la couleur de la merveille}

4 Le conte est un récit situé dans un temps et un lieu indéterminés («Il était une fois »), alors que la légende - transcrite depuis plusieurs siècles comme récit historique - met en scène des personnages censés avoir existé, en des lieux dont le nom attesterait de l'ancienneté et de la vérité des faits racontés. Le conte est donc fiction assumée et plus encore lorsqu'il est « merveilleux » suscitant, au sens étymologique, l'étonnement voire la sidération par l'intermédiaire des personnages ou des objets surnaturels qu'il met en scène. Ainsi, les héros de ces récits étiologiques, pédagogiques ou symboliques peuvent tisser en une nuit un tapis magique, bâtir un château de verre, devenir oiseaux ou se rendre invisibles afin de résoudre une énigme, de franchir un obstacle ou de surmonter les épreuves nécessaires à la réussite d'une initiation. On peut aussi avancer que ces histoires mythiques permettent l'exploration d'un univers dépourvu des cloisons dont le monde réel est hérissé. Le conte parle d'une terre où tout palpite, où la pierre est vivante, où les animaux parlent, où les arbres donnent des conseils. Par l'intermédiaire de ces narrations, une perception émerveillée de leur environnement est offerte aux lectrices et aux lecteurs, et de fabuleuses plages de liberté se créent pour les auteurs qui réécrivent ces textes ayant traversé le temps et parfois l'espace. Chacune et chacun en retient un personnage (la bonne fée de "Cendrillon » ou le Chat botté), une scène imagée (la pomme empoisonnée offerte à Blanche-Neige), ou parfois une couleur (la blondeur de Boucle d'or).

$5 \quad$ Il est cependant plus aisé de retrouver dans ces récits des types de personnages précis ou des schémas communs - comme le fait Vladimir Propp, par exemple - que des gammes variées de couleurs. L'observation des titres des contes réécrits par les auteurs les plus célèbres tels que Perrault, les frères Grimm ou Andersen en attestent. Ainsi, parmi les onze contes publiés par Charles Perrault («Grisélidis », «Peau d'Âne », « Les souhaits ridicules", «La Belle au bois dormant», «Le Petit Chaperon rouge », «La Barbe bleue », «Le Maitre chat, ou le Chat botté », «Les Fées », «Cendrillon », « Riquet à la houppe », «Le Petit Poucet»), seuls deux titres font explicitement mention d'une couleur. Pour comprendre la spécificité de cette gamme chromatique, on peut se référer notamment aux recherches éclairantes de Michel Pastoureau sur le rapport des populations occidentales à la couleur au fil des époques. Dans l'ouvrage Une histoire symbolique du Moyen Âge occidental ${ }^{1}$ consacré, entre autres, à l'histoire et à l'archéologie des couleurs ainsi qu'à l'origine des armoiries et des drapeaux, il souligne combien la conception symbolique des couleurs et des images, des signes et des songes, loin de s'opposer à la réalité sociale, économique ou politique, est une des composantes essentielles des sociétés médiévales. La couleur, qui possède en général une signification précise, est également représentative d'un état d'esprit. L'héraldique, les 
coutumes vestimentaires (deuil, mariage, etc.) en sont des témoignages aujourd'hui encore, en particulier dans les sociétés qui ont conservé des traditions fortes. De ce fait, la couleur ne s'emploie pas à la légère dans le quotidien, et encore moins dans les œuvres d'art qu'il s'agisse de tableaux, de vitraux, ou de fables ou de contes.

6 Il faut noter aussi qu'à l'orée de la période au cours de laquelle les contes merveilleux furent largement narrés et transcrits en Occident, à partir notamment du XII ${ }^{\mathrm{e}}$ siècle et jusqu'au XVII e siècle, l'Église pour des raisons éthiques et liturgiques manifestait une méfiance, voire un rejet, à l'encontre de la couleur qui pourrait également expliquer sa relative rareté dans les contes.

La tradition chromophobique d'une partie de l'Église (Pastoureau, 2004a) pour la fin $\mathrm{du}$ Moyen Âge, reste largement dominante dans les milieux monastiques espagnols $\mathrm{du} \mathrm{XVI}^{\mathrm{e}}$ siècle. La couleur est ce qui cache, ce qui dissimule, ce qui travestit la réalité des choses, elle est du côté du masque, du déguisement et, au final, du diable. La fortune de l'expression "sous couleur de ", extrêmement présente dans les textes de l'époque, en est un reflet. Ce rejet est global et porte non sur l'interprétation symbolique de chaque couleur - comme par exemple le rejet du jaune en tant que signe de maladie - mais bien sur une contestation du coloré en tant que tel, le terme « coloré » étant régulièrement employé avec une connotation négative ${ }^{2}$.

Ce rejet de la polychromie influence durablement les conteurs et leur fait préférer le régime du blanc et du rouge, ou celui du blanc et du noir :

De la protohistoire jusqu'au XII ${ }^{\mathrm{e}}$ siècle, l'Occident a [...] vécu sur cette structure trichrome constituée par le blanc et ses deux contraires : le noir et le rouge. Non pas que les autres couleurs n'aient pas existé. Mais dès qu'il s'agissait de mettre en forme des systèmes symboliques d'oppositions ou de hiérarchies, tout s'ordonnait autour de ces trois couleurs polaires ${ }^{3}$...

La lumière solaire blanche célèbre un monde positif: corps diaphane des fées bénéfiques, des princesses fragiles à la beauté opalescente. Le rouge est la représentation du sang et du feu, qui peuvent être porteurs de valeurs positives aussi bien que négatives. L'obscurité de la nuit dissimulatrice représente un univers ou un personnage négatif, suppôt de Satan ou incarnation du mal, sorcière ou démon. La merveille s'exprimerait davantage par le contraste entre le noir et le blanc, la nuit et l'éclat du jour ou du soleil, le sale et le blanc pur que par un large spectre de couleurs. Le choix d'une couleur précise (le chaperon " rouge », la barbe "bleue ») pourrait dans ce cas être essentiellement symbolique et appeler l'interprétation.

7 Lorsque l'on considère le conte " Peau d'Âne » réécrit par Perrault, on peut y observer, $\mathrm{au} \mathrm{XVII}{ }^{\mathrm{e}}$ siècle encore, cette " couleur de la merveille». Les folkloristes contemporains rattachent «Peau d'Âne» au cycle de Cendrillon par le motif des trois robes ou par celui de l'épreuve finale. On lui attribue le même numéro 510 dans la classification internationale Aarne-Thompson-Uther ${ }^{4}$ et on retrouve des formes de ce conte non seulement en Europe mais aussi en Afrique, en Asie, en Amérique (incluant des versions québécoise et guadeloupéenne). Pour l'anthropologue française Nicole Belmont, qui retrace la fortune incroyable de ce conte dans Sous la cendre: figures de Cendrillon, cette histoire trouverait son origine au Moyen-Orient, même si sa première forme écrite, datant $\mathrm{du} \mathrm{XI}^{\mathrm{e}}$ siècle, serait chinoise ${ }^{5}$. Elle propose de lire la nébuleuse du cycle de Cendrillon dont fait partie Peau d'Âne « comme un itinéraire psychique, celui des filles face à leur mère [...], qui doit aboutir à l'acquisition de la féminité, à la conscience de sa propre identité, et à la reconnaissance de celle-ci ${ }^{6} »$. Perrault, pour sa version du conte, s'est inspiré de plusieurs sources: celle des écrivains italiens Giovanni Francesco Straparola, dont la Doralice s'enfuit en Angleterre dans un coffre afin d'échapper à son 
père incestueux, et celle de Giambattista Basile qui fait se transformer sa Preziosa en ours pour qu'elle échappe au sien. Dès 1781 , une version en prose du conte est publiée et attribuée à Perrault. Cette version apocryphe désigne la bonne fée du conte original de Perrault sous le nom de "fée des Lilas » et atténue, de manière significative, la culpabilité du père encore plus que ne le fait Perrault, tout d'abord par la requête de la reine qu'à sa mort le roi prenne une nouvelle épouse et, ensuite, par les conseils que donnent au roi ses ministres d'épouser sa propre fille. Comme le conte de 1694, cette version apocryphe connut de nombreuses éditions, notamment au XIX siècle, et fut adaptée à plusieurs reprises, en particulier dans l'Alphabet des contes de fées, publié en 1866 , à la fin duquel la princesse demande pardon à son père incestueux pour s'être enfuie!

On connaît la version de Perrault : un roi veuf, sans descendance masculine, est pressé par ses conseillers de se remarier. Pour respecter le serment qu'il a fait à son épouse, ce roi ne peut épouser en secondes noces qu'une femme plus belle que la défunte reine. Or, la seule personne qui réponde à cette exigence est sa propre fille à qui il fait part de sa décision. Effrayée, la jeune princesse demande assistance à sa marraine. Suivant les conseils de la fée, la princesse exige de son père trois robes irréalisables, mais il réussit à les faire coudre. La jeune fille lui demande alors la peau d'un âne précieux qui fait de l'or et contribue à l'immense fortune du monarque'. Le roi y consent. Au comble de l'effroi, la princesse, déguisée en souillon et couverte de la peau de l'âne, se sauve alors dans une ferme. Un prince passant par-là tombe amoureux d'elle. De retour dans son royaume, il n'a de cesse de la retrouver. Ses efforts semblent vains jusqu'au jour où Peau d'Âne lui fait parvenir une bague dans un gâteau. Il la reconnaît grâce à cet anneau qu'elle est la seule de toutes les prétendantes au mariage promis par le prince à pouvoir enfiler à son doigt.

Perrault propose une version versifiée de l'histoire. En ce qui concerne le traitement des couleurs, il apparaît qu'il joue sur les valeurs symboliques qui leur sont le plus souvent associées et sur l'esthétique d'une époque. Dès le début du texte est célébrée la «magnificence» d'un lieu riche et heureux à la fois. L'or, symbole de puissance, de beauté et de lumière, en est la couleur essentielle. Le paradoxe vient cependant du moyen de production de cet or crotté par un âne magique. L'or et l'ordure, la lumière et son contraire sont présents ensemble au début du récit annonçant une suite funeste :

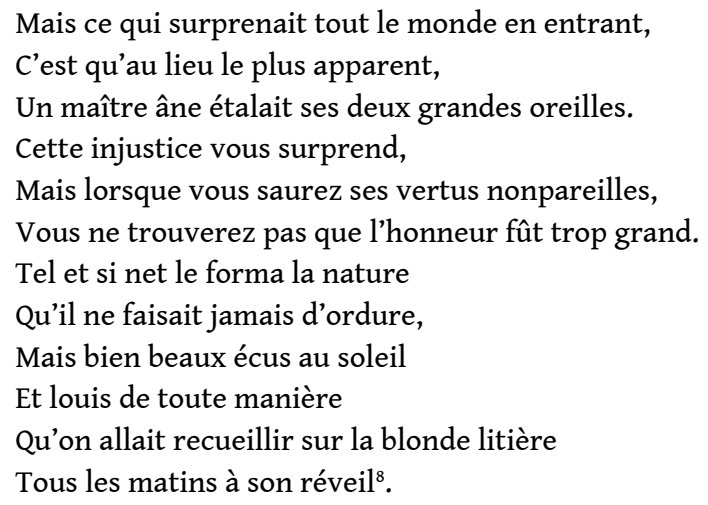

10 L'éclat de l'or, de l'argent et des pierreries sont un des motifs essentiels du conte. Les louis produits par l'âne magique, la paille où sont recueillies les pièces d'or, la chevelure blonde de Peau d'Âne, son teint vermeil et ses blanches mains, le jonc d'or de son anneau, les diamants et les pierres précieuses dont sont constellées les robes offertes par son père à la jeune fille, le « corps d'argent » (v. 404) qui constitue l'un de 
ses bustiers, déclinent, au cœur du texte, les différents états de la lumière du soleil et de la lune. La grotte «[d]e nacre et de corail richement étoffée » (v. 127) qui sert de logis à la marraine de la princesse ajoute des nuances précieuses à l'univers luxueux mis en place dans ce cadre. On notera l'absence quasi-totale de la couleur verte seulement suggérée par l'émeraude de la bague que Peau d'Âne cache dans le gâteau qu'elle confectionne pour le prince. Il semble que la pierre précieuse soit utilisée dans ce cas davantage pour son éclat que pour sa couleur proprement dite. Ces termes de couleur comportent tous l'idée de brillance ou du jeu de la lumière. L'amour de la lumière commun à toutes les civilisations est particulièrement manifeste dans l'Occident médiéval. L'éclairage défectueux abrégeait les veillées, rendait plus longues les nuits et plus désirable le retour du soleil. La survenue de la lumière du matin au printemps est célébrée par les trouvères dans la poésie et associée à la naissance du sentiment amoureux. Pour compenser les heures grises de l'existence et pour écarter l'obsession de la nuit, le Moyen Âge semble aussi avoir le goût des couleurs éclatantes comme le souligne la polychromie des vitraux, des étoffes, des bijoux, de l'héraldique et même des parures militaires. Enfin, la lumière source de beauté, de noblesse est également à considérer sur un plan mystique. Que l'on songe par exemple au Cantique $d u$ soleil de François d'Assise, au XIII ${ }^{e}$ siècle, où le Créateur est assimilé à l'astre du jour.

L'une des robes de la princesse cependant ne se contente pas de briller de tous ses feux, elle a la couleur du ciel, de l'azur : elle est bleue.

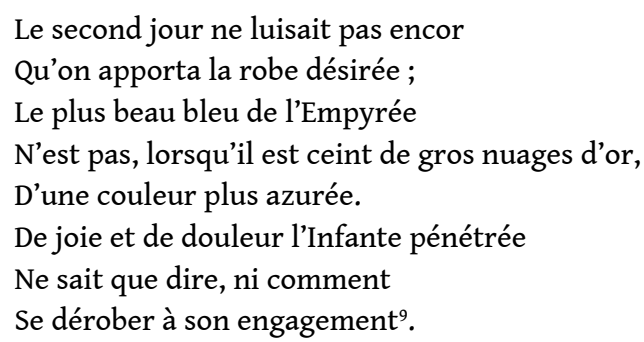

Dans son livre Bleu. Histoire d'une couleur, Michel Pastoureau ${ }^{10}$ explique que, dans l'Antiquité, le lexique des bleus est, en latin, imprécis et instable. Le bleu change de statut au XI ${ }^{\mathrm{e}}$ siècle : religieux et marial, il éclate dans les vitraux gothiques ${ }^{11}$ avant d'entrer en politique : les armoiries familiales des Capet (fleurs de lys sur fond d'azur) deviennent l'emblème du roi de France vers 1130. Le rouge reste impérial et papal, mais le bleu devient royal : c'est la couleur du légendaire roi Arthur ${ }^{12}$. La robe azurée de Peau d'Âne manifeste ici l'appartenance de la jeune fille - dont on dit qu'elle a aussi les yeux bleus - à une société aristocratique tout en étant le gage de sa pureté et de sa virginité.

L'envers de ce monde brillant, noble, pur n'est pas précisément un espace nocturne ou sombre. Même lorsque la nuit est évoquée, au moment où Peau d'Âne demande à son père une robe couleur de lune, c'est la « vive clarté » de l'astre d'argent rivalisant avec l'éclat des étoiles qui est essentiellement mis en valeur. À trois reprises seulement dans le texte, l'adjectif "noir» est employé. Il qualifie d'abord la gardeuse d'oies dont l'apparence produit effroi et dégoût, puis à la fin du texte les étrangers maures, touche exotique du cortège final, dont la laideur, de la même façon, effraie les enfants :

ô ciel ! Madame, lui dit-on,

Cette Peau d'Âne est une noire taupe

Plus vilaine encore et plus gaupe

Que le plus sale marmiton.

[...]

Mais lorsqu'elle tira de dessous sa peau noire 
Une petite main qui semblait de l'ivoire

Qu'un peu de pourpre a coloré,

Et que de la bague fatale,

D'une justesse sans égale,

Son petit doigt fut entouré,

La cour fut dans une surprise

Qui ne peut pas être comprise ${ }^{13}$.

Il s'agit de mettre, face à la magnificence, un univers de la souillure, de la saleté, de la pauvreté et de l'étrangeté. Aux couleurs de la merveille, lumière et azur, s'opposent les nuances suggérées, bises ou grisâtres, de la crasse et du dénuement, ou noires de la différence. La princesse fugueuse cachée sous une immonde peau d'âne est métamorphosée en une repoussante pauvresse. L'allusion à l'ordure laisse imaginer une palette variée de teintes répugnantes :

L'infante cependant poursuivait son chemin,

Le visage couvert d'une vilaine crasse,

À tous passants elle tendait la main,

Et tâchait pour servir de trouver une place ;

Mais les moins délicats et les plus malheureux

La voyant si maussade et si pleine d'ordure,

Ne voulaient écouter ni retirer chez eux

Une si sale créature ${ }^{14}$.

Le rouge, enfin, peu cité dans le récit, est synonyme de souffrance lorsqu'il est suggéré. C'est le feu néfaste de la passion dont l'«âme [du père incestueux] était embrasée » (v. 552).

13 Charles Perrault semble vouloir s'en tenir à la palette simple et éclatante qui est celle des contes merveilleux dans la pure tradition médiévale: des couleurs élémentaires, des systèmes symboliques anciens. Cependant, si l'on y regarde de près, ces couleurs apparaissent à des endroits significatifs du récit et sont loin d'être purement ornementales. Ainsi, l'origine similaire de l'or et de l'ordure, la production de l'éclat lumineux des robes conjuguée à de sombres intentions, le travestissement repoussant de Peau d'Âne qui, lorsque la jeune fille s'en dépouille, révèle la beauté dans son éclat et sa pureté, sont autant d'oppositions savamment orchestrées dans le conte pour dire non seulement la couleur de la merveille, mais pour exprimer toute l'ambiguïté de l'amour d'un père aux intentions incestueuses. Le déploiement apparemment classique des couleurs de la merveille : or, argent, azur, feux des pierreries, qui rencontrent la boue des déjections et la saleté tout comme le rouge du feu de la passion coupable, souligne une très grande maîtrise d'écriture propre à évoquer un conte de fées dans la plus pure tradition, tout en attirant l'attention du lecteur sur les perversions d'un système apparemment idéal. La magnificence produit en même temps que la lumière dorée ou blanche qui éclaire un monde parfait les teintes grisâtres et abjectes d'un univers corrompu et souillé.

\section{Peau d'Âne de Jacques Demy : la merveille de la couleur}

Les contes ont trouvé, avec la médiation du cinéma, un formidable outil pour mettre en scène la merveille. À l'orée du $\mathrm{xx}^{\mathrm{e}}$ siècle, Georges Méliès, contrairement aux frères Lumière, réalisateurs de films réalistes, a souhaité adapter des contes à l'écran. L'intérêt pour un auteur de cinéma était multiple. D’une part, il avait accès 
gratuitement à une réserve d'histoires libres de droits, d'autre part, il pouvait donner libre cours à sa fantaisie et distraire son public par l'intermédiaire de ces histoires patrimoniales et intemporelles dont la féerie se prête à toutes les interprétations. L'animation cinématographique allait bientôt prouver qu'elle pouvait parfaitement se mettre au service de la fantasmagorie du conte et donner ainsi à l'imaginaire le moyen de se déployer grâce aux performances de la technique. Dès 1937, les studios Disney produisent un dessin animé, Blanche Neige et les sept nains, qui remporte un immense succès et sera suivi, du vivant de Walt Disney, de seize autres films d'animation dont la plupart sont adaptés de contes occidentaux. Rien n'a endigué depuis lors le succès de ces films. Qui n'a en mémoire la remarquable adaptation que Jean Cocteau fit, en 1946, de l'œuvre de Madame Leprince Beaumont, La Belle et la Bête? Plus récemment, le cinéaste américain Tim Burton sut s'inspirer de ce patrimoine littéraire pour réaliser Edward aux mains d'argent en 1991.

Le cinéma et le dessin animé ont rendu possible à l'écran, par l'intermédiaire des trucages, la transformation de la grenouille en prince ou de la citrouille en carrosse. Jacques Demy semble en avoir pris toute la mesure lorsqu'il adapte "Peau d'Âne » de Charles Perrault pour le grand écran. Contemporain de la Nouvelle Vague, il reconnaît ce que son art doit à Méliès et à ses "transformations à vues ». Il s'en inspire lorsqu'il porte à l'écran les métamorphoses magiques qui abondent dans «Peau d'Âne ». Ainsi, on voit Peau d'Âne quitter ses habits de souillon en un clin d'œil pour redevenir princesse dans sa cabane de souillon, ou se dédoubler lorsqu'elle cuisine au simple moyen du champ/contrechamp. Lors de son évasion du château paternel, son carrosse devient charrette grâce au raccord d'un plan à l'autre. Le cinéaste utilise le ralenti combiné à une marche avant ou arrière pour passer d'un monde à l'autre. Il fait défiler la pellicule à l'envers afin de donner l'illusion, par exemple, que les bougies s'allument toutes seules.

16 Lorsque le réalisateur décide d'adapter l'œuvre au cinéma, il a déjà sept courts métrages et cinq films à son actif : Lola en 1961, La Baie des Anges en 1963, Les Parapluies de Cherbourg en 1964, Les Demoiselles de Rochefort en 1967 et Model Shop en 1968, et il est clair qu'il possède une bonne maîtrise $d u 7^{\mathrm{e}}$ art. Lors de mises en scène colorées, vivantes, musicales et perfectionnistes, il fait défiler une pléiade de personnages, marins, forains, fées, princes et princesses, ouvriers, jumelles excentriques, artistes rêveurs qui se livrent, dans des villes portuaires ou dans des forêts de contes, par exemple, à des chassés-croisés amoureux dignes des pièces de Marivaux. Il désire tous ces films "liés les uns aux autres", au service de son univers intérieur qui puise dans son enfance. L'imaginaire y a toujours raison de l'impossible. Il a recours aussi à sa bibliothèque intérieure où se côtoient diverses influences. Quoique fidèle à la ligne essentielle du conte, le film est ainsi une adaptation double du conte «Peau d'Âne » de Charles Perrault et de la version en prose anonyme, publiée en 1781 à Paris chez Lamy et illustrée au XIX siècle par Gustave Doré (édition Hetzel, 1862). Le cinéaste fait aussi de nombreuses allusions à d'autres contes célèbres de Perrault: lorsque Peau d'Âne après sa fuite arrive dans une ferme, tous les personnages sont immobiles, figés dans leur activité comme les acteurs endormis de « La Belle au bois dormant » victimes d'un mauvais sort. À un autre moment, le bal des chats et des oiseaux, organisé par la Reine rouge, doit accueillir le marquis de Carabas, héros du «Chat botté ».

17 La convocation de ces nombreuses citations cinématographiques et littéraires souligne l'importance que Jacques Demy accorde à la réussite de la fabrication de ce film. Le défi 
est d'envergure car il faut se montrer à la hauteur des référents cités, tout en ne trahissant pas les rêves de l'enfant-lecteur devenu réalisateur. Par l'intermédiaire de Jean Marais dans le rôle du roi, père de Peau d'Âne, ainsi que des citations directes aux costumes et aux décors du chef-d'œuvre de Jean Cocteau, La Belle et la Bête, il rend un vibrant hommage au poète. Mais peut-être plus encore que les trucages cinématographiques ou l'héritage de prédécesseurs inspirés, c'est l'invention du technicolor qui, autorisant toutes les fantaisies polychromes et élargissant considérablement la palette de la "merveille", va donner l'occasion au cinéaste de tenter toutes sortes d'expérimentations dans Peau d'Âne. En 1970, de retour des ÉtatsUnis, Jacques Demy est au sommet de son art. Il s'est enrichi, lors de son périple dans le Nouveau Monde, de l'extraordinaire culture cinématographique de ce pays. Il a aussi fréquenté des artistes de tous bords en pleine vogue hippie. Le film en est marqué, se présentant comme un voyage anachronique qui célèbre aussi bien Andy Warhol que Walt Disney. Il s'inspire notamment du dessin animé Blanche-Neige et les sept nains. Pour autant, il ne s'agit pas pour Demy de faire un film palimpseste et la créativité y a une part importante par l'intermédiaire de la musique de Michel Legrand, mais aussi et peut-être surtout par le traitement des décors, des costumes et de la couleur.

Suite à des problèmes financiers, Demy se voit obligé de renoncer à la mise en scène sophistiquée dont il rêvait lors des premières moutures du scénario, en mars 1969, qui prévoyait des plans mobiles et complexes avec usage de grues et travellings. Il est contraint, pour les mêmes raisons, de se passer des services du décorateur Bernard Evein qui a collaboré à de précédents films. Il le remplace par Jim Leon, rencontré à San Francisco. Ce créateur fait se croiser pop art et conventions esthétiques du conte dans une débauche de couleurs et une profusion de matières. Il signe l'affiche du film, témoin du courant psychédélique qui traverse l'époque, souvenir des Renaissance fairs américaines moins attachées au passé historique qu'à sa recréation imaginaire.

Lorsque Jacques Demy m'a demandé de lui envoyer des dessins de moi, je lui en ai envoyé deux : un papillon et un dessin érotique.

Puis nous nous sommes rencontrés et c'est comme ça que j'ai imaginé 28 maquettes pour les décors de son film Peau d'Âne.

Je suis un supporter inconditionnel de l'art onirique du $\mathrm{xIX}^{\mathrm{e}}$ siècle et de tous les siècles de l'homme. Je pense que seul un impuissant de l'esprit peut croire que le conte de fée est un domaine réservé à l'enfance ou est une fuite de la réalité matérielle. C'est plutôt une autre facette d'un même phénomène. Le mythe, c'est l'autre réel ; peut-être le vrai ${ }^{15}$.

L'inventivité du décorateur n'est jamais prise en défaut. Si l'ornementation est relativement simple, circonscrite aux vitraux ou à des éléments de décors précis dans de vastes espaces intérieurs : lits, paravent, trônes, miroir, etc., il sait faire feu de tout bois. Deux cerfs factices, trouvés dans le hall de Chambord, deviennent les gardiens d'un lit planté au milieu de la chambre, leurs bois se confondant avec la végétation luxuriante du lieu. Ils s'ajoutent au riche bestiaire du film mêlant l'animal et le végétal.

Mais c'est surtout en optant pour des couleurs vives et tranchées comme les aimait Andy Warhol, que Jim Leon sert le mieux le propos du film. Dans les premières scènes règne l'ambiance bleue - le bleu Klein - et glaciale d'une forteresse féodale fermée sur elle-même. La princesse et son père sont vêtus de cette couleur froide et servis par des pages aux visages peints en bleu. À la cour du roi, les chevaux, les habits, les meubles, la tranche des livres ou les plumes du paon qui parade dans la cour du château sont bleus. Depuis la mort de la reine en hiver par un jour de neige, la vie semble s'être retirée du palais. Consciemment ou non, décorateur et metteur en scène utilisent, comme au 
Moyen Âge, la couleur bleue pour symboliser le deuil. Dans le conte de Perrault, aucune couleur n'évoque cette période sombre de la vie du roi passée quasiment sous silence - au XVII ${ }^{\mathrm{e}}$ siècle la mort des êtres jeunes n'a rien d'exceptionnel. Dans le film de Demy, au contraire, la musique et la couleur insistent sur la longueur et la tristesse du deuil auquel les contemporains du cinéaste sont devenus très sensibles. La couleur est ici clairement du côté de l'émotion que l'image sert sans modération. En outre, la couleur bleue n'est pas sans évoquer l'expression « avoir du sang bleu » qui signifie appartenir à une noble lignée forcément marquée par la consanguinité. Ce que ne renie pas le Roi bleu lorsqu'il propose à sa fille de l'épouser dans ce royaume clos sur lui-même et endogame, à l'image de son château tourné vers la cour intérieure. Enfin, cette teinte obsédante rappelle un autre conte de Perrault, «La Barbe bleue », dont le cruel héros a assassiné sans remords ses épouses trop curieuses qui faisaient montre d'un début de révolte et de volonté d'indépendance en lui désobéissant.

Pour le château du prince charmant, murs et mobilier, Jim Leon a choisi, au contraire, respectant le souhait de Demy, un carmin chaleureux. On donne, dans ce décor avenant, des fêtes somptueuses. Le prince est vêtu de rouge et même ses fiers destriers sont peints de cette couleur. Tout se distribue autour d'une idée forte, une division chromatique impérative: une famille royale en rouge et l'autre en bleu. Une fois encore, se traduit explicitement par la couleur les mots et les idées portés par le conte original. Les codes symboliques sont transparents. Si, après la mort de sa mère, la princesse a grandi, délaissée par son père fou de chagrin, dans un monde privé d'amour, elle éprouve le feu de la passion dès sa rencontre avec le prince qui en est l'incarnation. En outre, le rouge du royaume, où Peau d'Âne se réfugie pour échapper à la volonté de son géniteur et de son roi, pourrait représenter aussi le feu de la colère et de la révolte qui a fini par animer la jeune fille refusant de se plier à la volonté perverse de son père incestueux. Le prince, lui-même, ne s'oppose-t-il pas aux obligations de sa condition en s'enquérant puis en tombant amoureux d'une souillon dont il ne devrait pas même remarquer l'existence? Mai 68 encore tout proche a donné l'occasion aux jeunes générations de faire la révolution contre les figures de père castratrices et oppressantes, qu'elles soient familiales ou politiques.

La fée marraine, vêtue de mauve, synthèse de la couleur froide et de la couleur chaude, tente une transition entre ces deux univers qu'elle se donne beaucoup de mal à harmoniser, en inventant des épreuves propres à détourner le père de la princesse de ses mauvaises intentions. Déclinant, dans trois demandes de création de robes impossibles, de subtiles variations sur la lumière, elle met au défi le mal de s'affronter à l'éclat du jour, de la lune et du soleil. Une gageure aussi pour le réalisateur qui s'adresse à Agostino Pace, costumier de théâtre, pour les dessiner, tandis que Gitt Magrini - costumière d'Antonioni et de Bertolucci - se charge de les fabriquer en Italie. Couleur du temps, de la lune et du soleil, les tissus sur lesquels courent les nuages et s'allument les étoiles laissent sans voix même la fée des Lilas qui ne pensait pas «que cela fût possible». Pas moins de dix robes sont prévues, contre quatre costumes différents pour le prince. Le spectateur ébloui gardera en mémoire ces extraordinaires tenues pailletées, moirées, somptueusement brodées de pierreries, largement déployées en corolle autour de la taille fine de Catherine Deneuve d'une blondeur irréelle. Elles symbolisent sans doute de la façon la plus évidente la conception de la couleur du merveilleux selon Demy: «On doit sentir les matières des tissus, écrit-il dans ses notes préparatoires. Brocarts, paillettes, diamants, voiles, cristaux, perles, etc. 
Simples mais de couleurs, illustrations enfantines plus proches de Disney que du bon goût, ou plus Disney que [Gustave] Doré16. .

Finalement, Leon et Demy choisissent le blanc à la fin du film, pour faire le lien entre les personnages qui s'affichent dans les costumes de lumière de la grande scène du mariage. Le blanc, addition de toutes les couleurs du prisme, symbole dans les contes merveilleux, au même titre que l'or du soleil, de la perfection et/ou de l'innocence atteinte ou retrouvée. C'est, chez Perrault, la main d'ivoire de Peau d'Âne nettoyée de sa crasse, la splendeur éclatante de sa chevelure dorée débarrassée de la peau malodorante de l'âne mort et, dans le film de Demy, la représentation de l'amour sublimé et du rêve de l'harmonie universelle. Les couleurs du merveilleux chez Demy agissent non seulement comme le ferait une illusion magique, mais aussi et surtout comme un révélateur. L'habillage du conte ne doit pas faire oublier l'essentiel de ce qu'il dit. Toutes les couleurs se conjuguent à la fin du film dans le cortège arc-en-ciel du mariage. Par l'intermédiaire de la robe blanche de Peau d'Âne qui rassemble dans le faisceau de la lumière toutes les couleurs, est célébré, pour résoudre les malheurs et les perversions du monde, non seulement l'amour passion du couple mais aussi l'amour solaire universel avec un grand $\mathrm{A}$ auquel croyaient les hippies.

Jacques Demy n'est pas Walt Disney, cependant. Il ne souhaite pas entraîner ses spectateurs sur les chemins de l'illusion, les faisant adhérer au monde idéal que permet d'inventer le cinéma par l'intermédiaire d'images qui subliment artistiquement la réalité. Il a soin d'attirer l'attention sur la fabrication de l'univers coloré qu'il met en place, et la mise en scène de l'illusion est constamment visible. Toute l'esthétique du film semble à la lisière du second degré. Il y a dans le luxe même de l'apparat un toc assumé, une fausseté revendiquée que ne démentent pas les trucages. Cette distanciation voulue se remarque par l'intervention de l'humour et des anachronismes évidents : la fée des Lilas, par exemple, a le téléphone et se déplace en hélicoptère pour la scène finale au cours de laquelle le défilé anachronique des invités de la noce a des accents baroques voire rococo. Le merveilleux doit agir non comme le ferait une illusion magique mais plutôt comme un révélateur au sens propre aussi bien que figuré. Il ne faut pas se tromper, nous sommes bien ici dans le monde du conte, et la profusion de couleurs fausses (un cheval ne peut pas plus être bleu ou rouge qu'un âne ne peut crotter de l'or) rappelle ostensiblement que, dans la réalité, on ne peut pas aussi aisément résoudre les problèmes - aussi douloureux notamment que l'inceste - par un coup de baguette magique que ce soit celui d'une fée ou celui d'un metteur en scène talentueux.

Lorsque Charles Perrault réécrit Peau d'Âne s'en tenant à un sobre "code couleur " selon la tradition médiévale, ou lorsque Jacques Demy met le conte en scène s'autorisant des fantaisies colorées psychédéliques selon une conception moderne, ils nous donnent à voir en toute connaissance de cause leur version d'un conte merveilleux.

Chez l'auteur du XvII siècle, la dominante dorée qui nimbe toute l'histoire voisine manifestement avec l'ordure. La lumière et la saleté se côtoient pour rappeler que toute société, aussi idéale paraisse-t-elle, possède non seulement son revers ténébreux mais le développe souvent conjointement à son avers lumineux, l'un naissant de l'autre. Le réalisateur $\mathrm{du} \mathrm{xx}^{\mathrm{e}}$ siècle souligne, par l'intermédiaire d'une esthétique cinématographique à la lisière du second degré, qu'il serait aussi vain de se laisser prendre à l'explosion de couleurs se résolvant dans le prisme d'une lumière blanche 
surnaturelle qu'à la magie d'un monde idéal - aussi éphémère que truqué - où les princesses épousent, lors de fêtes grandioses pleines de rires et de musique, les princes dont elles sont amoureuses.

Dans les deux versions du conte, éloignées de trois siècles, l'utilisation de la couleur attire l'attention du lecteur ou du spectateur, respectivement contemporain de l'écrivain ou du cinéaste, sur les pièges de l'illusion de l'univers merveilleux mis en scène tout en les amenant, par l'intermédiaire d'une symbolique diffuse ou évidente, à s'interroger sur l'état du monde réel dans lequel ils vivent. L'analyse de la palette chromatique des deux auteurs révèle non seulement une manière de faire par la mise en récit d'une poétique de la merveille, mais aussi une manière d'être par la façon dont ils souhaitent, au moyen de l'imaginaire, rendre compte des différentes facettes, y compris les plus sombres ou les plus cachées, du monde qui les entoure.

\section{NOTES}

1. M. Pastoureau, Une histoire symbolique du Moyen Âge occidental, Paris, Seuil, coll. « La Librairie du $\mathrm{xxI}^{\mathrm{e}}$ siècle $", 2004$.

2. A. Roullet, «Le Refus de la couleur : corps et mystique en Espagne au XVI ${ }^{\mathrm{e}}$ siècle », Corps 2007/2, $\mathrm{n}^{\circ} 3$, p. 41-47.

3. M. Pastoureau, "Et puis vint le bleu...", Europe, $\mathrm{n}^{\circ} 654$, octobre 1983, p. 43. Voir aussi

M. Pastoureau, L'Hermine et le sinople. Études d'héraldique médiévale, Paris, Le Léopard d'Or, 1982.

4. ATU 510A «Cendrillon » et ATU 510B « La robe d'or, d'argent et d'étoiles (Peau d'Âne) ».

5. Sous la cendre: figures de Cendrillon, anthologie établie et postfacée par N. Belmont et É. Lemirre, Paris, José Corti, coll. « Merveilleux », 2007, postface, p. 170.

6. Ibid., p. 12.

7. Il faut noter à ce propos que la version de Perrault est la seule qui évoque l'âne crottant de l'or, cet épisode appartenant à un autre conte, La Serviette, l'âne et le bâton (ATU 563). Mais cette contamination est ingénieuse puisque le sacrifice de cet âne prodigieux prouve que le roi est prêt à tout pour satisfaire sa passion.

8. C. Perrault, Contes, éd. T. Gheeraert, Paris, Honoré Champion, coll. «Champion Classiques", 2012, v. 47-58, p. 141-142.

9. Ibid., v. 158-165, p. 147.

10. M. Pastoureau, Bleu. Histoire d'une couleur, Paris, Seuil, 2000.

11. Ibid., p. 52.

12. Ibid., p. 60.

13. C. Perrault, ouvr. cité, respectivement v. 388-391, p. 156 et v. 500-507, p. 161.

14. Ibid., v. 266-273, p. 151.

15. Voir <jim-leon.net/peaudane.shtml>. Peau d'Âne doit beaucoup au peintre britannique Jim Leon, auteur de l'affiche du premier concert des Pink Floyd en France.

16. Zoom sur « Peau d'Âne, Demy et le Merveilleux », Cinémathèque française, 2013. 


\section{RÉSUMÉS}

L'observation de la réécriture de certains contes permet de mettre en évidence les liens qu'entretiennent le merveilleux et la couleur par l'intermédiaire de personnages, d'un bestiaire, d'objets et/ou de costumes magiques, créant, au fil du récit, un univers coloré, expression d'un genre comme d'une époque et d'un style. Lorsque Jacques Demy met en scène le conte Peau d'Âne, en ayant recours à une gamme chromatique étendue sans que Perrault ou ses prédécesseurs n'en ait fait explicitement mention dans le texte, sa lecture dévoile, d'une part, à quel point l'inventivité du cinéaste est stimulée par le récit merveilleux et, d'autre part, les ressources sémantiques indicibles du texte.

Nous essaierons, en analysant cette réécriture inspirée du cinéaste qui a rendu Peau d'Âne universellement célèbre, de voir ce qui, dans le texte, a pu susciter cette mise en couleur du conte initial, avant de tenter de cerner la symbolique et la poétique de ces couleurs dans le récit comme dans le film.

When Jacques Demy staged Peau d'Âne, using an extensive chromatic range without Perrault or his predecessors having made explicit mention of it in the text, his reading reveals how the filmmaker's inventiveness is stimulated by the fairy tale and the semantic resources of the text. We will try to explain what may have inspired this colour-setting of the initial tale and then we try to identify the symbolism and poetics of these colours in the fairy tale as well as in the film.

\section{INDEX}

Keywords : fairy tale, movie, marvel, chromatic range, symbolism, rewriting

Mots-clés : conte de fées, cinéma, merveilleux, gamme chromatique, symbolisme, réécriture

\section{AUTEUR}

\section{DANIĖLE HENKY}

Université de Strasbourg, Configurations littéraires EA 1337

daniele.henky@wanadoo.fr 